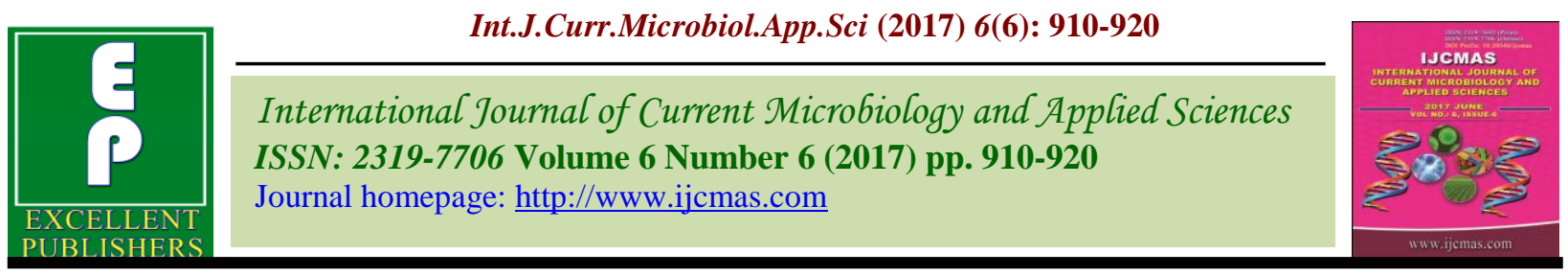

Original Research Article https://doi.org/10.20546/ijcmas.2017.606.107

\title{
Determine Physiological Traits Associated with Flowering Stage Drought Tolerance in Lowland Rice (Oryza sativa L.) Genotypes
}

\author{
A.K. Singh and A.K. Mall* \\ ICAR-Indian Institute of Sugarcane Research, Lucknow-226 002, India \\ Centre of Advance Studies in Plant Physiology, NDUA\&T, Kumarganj-224 229, India \\ *Corresponding author
}

\section{A B S T R A C T}

\begin{tabular}{l} 
K e y w o r d s \\
Reproductive stage \\
drought stress, \\
Rice, Morpho- \\
physiological traits, \\
Variability, \\
Correlation, \\
Path coefficient. \\
Article Info \\
\hline $\begin{array}{l}\text { Accepted: } \\
\text { 17 May } 2017 \\
\text { Available Online: } \\
\text { 10 June } 2017\end{array}$ \\
\hline
\end{tabular}

\section{Introduction}

Breeding rice for drought-prone conditions has had less success than breeding for favorable irrigated environments. There is a lower return on plant breeding for lower yielding upland environments, compounded by a more costly and slower uptake of new varieties. The plant breeding process for drought adaptation can be made more efficient when traits other than yield are added to the selection process. Eastern Uttar Pradesh share only $2.76 \mathrm{~m}$ ha area out of total 5.8 ha area under rice which an average yield of $1.42 \mathrm{t} \mathrm{ha}^{-1}$ and almost 15 per cent area is planted to rainfed upland. Rainfall pattern of this region is erratic and limited to short period, resulting in drought spells of 1-3 weeks at either seedling/vegetative and anthesis stages depending on the time of rainfall. Terminal drought is recurring feature in this region which is detrimental to rice yield.

Drought is a metrological term involving rainfall deficit and shows variation in 
intensity, duration and occurrence annually. Drought resistance is the genetic term used to cover a range of mechanisms whereby plants withstand periods of dry weather. It includes drought escape and drought tolerance with high or low tissue water potential. Drought escape is characterized by rapid phenological development and developmental plasticity, which enables the plants to complete its-life cycle before the onset of drought.

A deep root system is considered as important component of drought resistance because it related to the plants ability. A number of physio-morphological characters have been suggested to confer drought resistance in rice.

Low root densities at depth are the main reason for the ineffective use of available moisture in deeper soil layers and welldeveloped root systems are often associated with dehydration avoidance of cultivars in upland condition (O'Toole and Chang, 1979; Yoshida and Hesegawa, 1982; Ekanayake et al., 1985; Lilley and Fukai, 1994).

Enormous amount of variability is exhibited by traditional cultivars grown under fragile environments indicating that native landraces embody unique tolerance strategies appropriate to specific growing condition. Therefore, present investigation was carried out to estimate the existing variability in population.

\section{Materials and Methods}

\section{Experimental sites, genotypes and years of screen}

The present investigation was carried out in wet season, during 2007 and 2008 at the Instructional Farm of Department of Crop Physiology, N. D. University of Agriculture \& Technology Kumarganj (Faizabad), U.P., India. The genotypes of upland rice (indica and japonica type) from different geographical regions were screened for drought tolerance. These genotypes responded well under severe drought conditions and displayed good drought score, recovery and early vegetative vigour, simultaneously, substantial yield also.

\section{Management of water stress}

The experiments were conducted with well defined protocol for water management under natural field conditions during wet season in both the years.

\section{Irrigated control (E1)}

The experimental field was left uncovered to receive natural rainfall. In addition to this, experimental plots were irrigated using well laid channels for supplying tube well water, as and when required, to maintain appropriate moisture levels as recommended for irrigated rice.

\section{Reproductive stage drought stress (E2)}

The experiment field was covered by constructing temporary rainout shelter at a height of 10-12 feet using polythene sheets to exclude any possibility of natural rainfall falling in the experimental plots with proper drainage channel. Care was taken to check the inflow or seepage of water from the adjoining areas by making adequate bunds around the experiment and covered with polythene in drought condition.

The heading stage drought was created by withholding the irrigation for 15 days up to 80 $\mathrm{K} \mathrm{Pa}$ at $0-15 \mathrm{~cm}$ soil profile and $60 \mathrm{~K} \mathrm{~Pa}$ at 30 $\mathrm{cm}$ soil depth. Plants were exposed for two weeks (60-80 KPa). Soil moisture content (SMC) during stress period was monitored through periodical soil sampling at $0-15,15-$ $30 \mathrm{~cm}$ soil depth. Drought was released by 
irrigation. Recovery was measured at 10th days after released of drought.

Genotypes were scored for leaf rolling and leaf drying at the peak stress period using the IRRI Standard Evaluation System (IRRI, 1996).

\section{Experimental design}

The genotypes were seeded and seedling establishment was done in dry beds and transplanting was done 21 days after seeding. Each genotype was transplanted in Randomized Block Design with three replications in a $3 \mathrm{~m}$ length row. Row spacing was $20 \times 15 \mathrm{~cm}$ and one seedling per hill was used. Recommended agronomic practices were followed. Pesticides and bird nets were used to protect the plants against pests. All other crop management practices were at the optimum level.

\section{Observation and evaluation}

Observations were recorded on five competitive plants of the middle row of each plot for yield and 18 morpho-physiological traits. The plant height $(\mathrm{PH})$ was measured from the base of stem i.e. surface of the ground upto the top of the panicle. Panicle length (PL) of 5 panicles of each replication was randomly measured with the help of meter scale at maturity.

The no. of sterile SG/P and fertile seed FG/P on five panicles, selected randomly from each treatment were counted. Number of Ear Bearing Tillers (EBT) per plant under each treatment was recorded by visual counting. 1000 seeds from each treatment were counted and weighed for assessing test weight (TW) in each treatment.

Harvest index (HI) was calculated as per formula of Beedle (1982). Dry weight of shoot, panicle and root was recorded after drought (TB at flowering). Roots were removed from the PVC pipes after 15 days of drought exposure $(60-80 \mathrm{~K} \mathrm{~Pa})$ and washed the roots with tap water and root lengths (RL) were taken. Roots were removed from the plants and washed with the help of tap water and finally roots volume (RV) was measured by measuring cylinder.

The relative water content (RWC) was determined by the method described by Weatherley (1965). Water potential (WP) of main shoot was measured by the pressure bomb (made in soil moisture equipment corp, santa Barbara, CA, USA) method. Leaf membrane stability index (MTS) is determined by using protocol describe by Saadalla et al., (1990).

The post anthesis decrease in culm dry weight relative to increase in panicle dry weight (ATR) was calculated by the formula of Reyniess et al., (1982). The relative contribution of $\mathrm{CHO}$ accumulated before flowering to grain $\mathrm{CHO}$ at harvest (ACR) was calculated by the formula of Yoshida and Ahn (1968).

The data of morpho-physiological and grain yield were analyzed by appropriate statistical analysis (Gomez and Gomez, 1984) using CropStat 7.2 (IRRI, 2009) programme. Phenotypic (PCV) and genotypic (GCV) coefficients of variation, heritability (broad sense), genetic advance as percentage of mean $(\mathrm{Ga})$, correlation and path coefficient were computed following Singh and Chaudhury (1985).

\section{Results and Discussion}

\section{Correlation coefficients}

The correlation coefficient, which provides symmetrical measurement of degree of 
association between two variables or characters, helps us in understanding the nature and magnitude of associations among yield and yield components. In the present study, genotypic correlation coefficients between different character pairs were generally similar in sign and nature to the corresponding phenotypic correlation coefficients.

However, genotypic correlations were higher in magnitude than respective phenotypic correlations between various characters. Similar observations in rice have also been reported by Bai et al., (1992).

None of the physio-morphological characters exhibited strong positive association at genotypic and phenotypic level in control condition. SG/P resulted into negative and significant association condition. In drought condition, SY, PL, TB at $\mathrm{M}$ and $\mathrm{TB}$ at $\mathrm{F}$ emerged with positive and highly significant phenotypic correlations along with high order positive genotypic correlations with GY/P.

The above results indicated that none of the morpho-physiological traits appeared as strong associates of grain yield in irrigated control condition, whereas four traits, namely, SY, PL and TB at maturity and TB at flowering were found to be strong associates of grain yield in E2.

The strong negative associations at phenotypic as well as phenotypic level of $\mathrm{SG} / \mathrm{P}$ in both conditions and MTS in E2 were recorded. The above observation appears logical as increase in the number of SG/P are likely to reduce yield, while increasing MTS may have negative effect on yield only under E2.

Strong positive association at genotypic and phenotypic level was also observed between RWC and EBT, WP and TB at maturity, PH and FG/P and SY and PL in E1. In E2, SY showed strong positive association with RWC, PL, HI, TB at maturity and TB at flowering. While, TB at flowering had positive association with $\mathrm{TB}$ at maturity and PL. Positive association of MTS with SG/P and RV was observed in E2.

Thus, number of physio-morphological character pairs exhibited strong positive association in control condition, while in E2, thirteen character pairs had strong positive association at phenotypic as well as genotypic level. The number of characters pairs exhibiting strong negative association at both levels in E1 and E2 were two and eleven, respectively.

The above discussion emphatically underlines the existence of markedly high number of strong positive or negative associations in drought stress than control condition.

It is interesting to note that water stress resulted into negative associations among physio- morphological characters and yield in E2 than the E1.

Grain yield exhibited significant and positive correlation with $\mathrm{PH}$, and PL at genotypic as well as phenotypic level. Positive relationship of GY with EBT, FGP, TW and HI was reported by Reddy et al., (1995). Leaf RWC was negatively correlated with leaf rolling and days to heading under stress.

Leaf during scores had negative correlations with yield and harvest index under stress biomass under stress was positively correlated with yield, spike let fertility, G/P per cent stress were positively correlated with relative yield under stress (Babu et al., 2003).

These correlations between plant water status indicators and plant phenology and production traits under stress in this study confirmed the earlier reports in rice (Blum et al., 1999). 
Table.1 Estimates of genotypic and phenotypic correlation between physio-morphological characters in rice genotypes in $\mathrm{E}_{1}$

\begin{tabular}{|c|c|c|c|c|c|c|c|c|c|c|c|c|c|c|c|c|c|c|c|}
\hline Character & $\begin{array}{c}\text { Correlation } \\
r_{s}\end{array}$ & WP & RWC & PL & FG/P & SG/P & EBT & SY & HI & TW & TB at $F$ & TB at $M$ & ATR & ACR & MT & $\mathbf{R L}$ & RV & RDW & GY \\
\hline \multirow[t]{2}{*}{ PH } & $\mathrm{r}_{\mathrm{g}}$ & 0.446 & 0.223 & -0.038 & 0.671 & -0.052 & 0.400 & 0.061 & -0.207 & 0.250 & 0.327 & 0.360 & 0.292 & 0.00 & 0.064 & 0.362 & -0.119 & 0.280 & 0.00 \\
\hline & $\mathrm{r}_{\mathrm{p}}$ & 0.373 & 0.188 & -0.018 & $0.550^{*}$ & -0.048 & 0.228 & 0.052 & -0.176 & 0.101 & 0.230 & 0.298 & 0.236 & 0.002 & -0.052 & 0.302 & -0.149 & 0.233 & -0.006 \\
\hline \multirow[t]{2}{*}{ WP } & $\mathrm{rg}_{\mathrm{g}}$ & & -0.336 & 0.028 & 0.468 & 0.163 & -0.425 & 0.160 & -0.394 & 0.793 & -0.071 & 0.616 & -0.261 & 0.056 & 0.119 & -0.107 & -0.105 & 0.401 & -0.075 \\
\hline & $r_{p}$ & & -0.355 & 0.026 & 0.435 & 0.154 & -0.311 & 0.160 & -0.320 & 0.443 & -0.062 & $0.589 * *$ & -0.250 & 0.057 & 0.077 & -0.109 & -0.103 & 0.398 & -0.074 \\
\hline \multirow[t]{2}{*}{ RWC } & $\mathrm{r}_{\mathrm{g}}$ & & & -0.296 & -0.099 & -0.059 & 0.839 & -0.258 & -0.253 & 0.381 & -0.163 & -0.157 & 0.101 & 0.266 & 0.153 & -0.251 & 0.101 & 0.113 & -0.446 \\
\hline & $r_{p}$ & & & -0.294 & -0.093 & -0.057 & $0.604 * *$ & -0.256 & -0.201 & 0.209 & -0.148 & -0.151 & 0.097 & 0.266 & 0.092 & -0.249 & 0.099 & 0.113 & -0.445 \\
\hline \multirow[t]{2}{*}{$\mathbf{P L}$} & $\mathrm{rg}_{\mathrm{g}}$ & & & & -0.146 & -0.329 & -0.387 & 0.534 & 0.330 & -0.140 & -0.110 & -0.055 & 0.214 & -0.228 & 0.566 & 0.095 & 0.073 & -0.005 & 0.205 \\
\hline & $\mathrm{r}_{\mathrm{p}}$ & & & & -0.132 & -0.315 & -0.293 & $0.526^{*}$ & 0.271 & -0.091 & -0.094 & -0.052 & 0.197 & -0.226 & 0.362 & 0.093 & 0.071 & -0.005 & 0.203 \\
\hline \multirow[t]{2}{*}{ FG/P } & $\mathrm{rg}_{\mathrm{g}}$ & & & & & 0.097 & 0.186 & 0.311 & -0.115 & 0.178 & 0.167 & 0.283 & 0.146 & 0.233 & -0.080 & 0.063 & -0.315 & -0.058 & -0.088 \\
\hline & $r_{p}$ & & & & & 0.097 & 0.155 & 0.294 & -0.128 & 0.138 & 0.152 & 0.264 & 0.134 & 0.218 & -0.016 & 0.058 & -0.296 & -0.051 & -0.082 \\
\hline \multirow[t]{2}{*}{ SG/P } & $\mathrm{r}_{\mathrm{g}}$ & & & & & & -0.154 & -0.294 & -0.162 & 0.244 & -0.285 & -0.199 & -0.938 & 0.193 & -0.181 & 0.100 & 0.039 & 0.542 & -0.576 \\
\hline & $r_{p}$ & & & & & & -0.093 & -0.286 & -0.101 & 0.118 & -0.272 & -0.186 & $-0.873 * *$ & 0.186 & -0.084 & 0.097 & 0.040 & $0.529^{*}$ & $-0.559^{*}$ \\
\hline \multirow[t]{2}{*}{ EBT } & $\mathrm{r}_{\mathrm{g}}$ & & & & & & & -0.494 & -0.004 & -0.057 & 0.157 & -0.320 & 0.571 & 0.111 & -0.305 & -0.167 & -0.016 & -0.267 & -0.113 \\
\hline & $r_{p}$ & & & & & & & -0.351 & -0.001 & 0.191 & 0.045 & -0.206 & 0.405 & 0.082 & -0.110 & -0.117 & -0.013 & -0.193 & -0.086 \\
\hline \multirow[t]{2}{*}{ SY } & $\mathrm{rg}_{\mathrm{g}}$ & & & & & & & & 0.009 & -0.169 & 0.029 & 0.073 & 0.114 & -0.056 & 0.459 & 0.121 & -0.373 & -0.349 & 0.430 \\
\hline & $r_{p}$ & & & & & & & & 0.002 & -0.081 & 0.019 & 0.075 & 0.106 & -0.055 & 0.300 & 0.120 & -0.368 & -0.348 & 0.427 \\
\hline \multirow[t]{2}{*}{ HI } & $\mathrm{rg}_{\mathrm{g}}$ & & & & & & & & & -0.813 & 0.298 & -0.123 & 0.267 & -0.177 & -0.411 & 0.396 & -0.123 & -0.022 & 0.255 \\
\hline & $\mathrm{r}_{\mathrm{p}}$ & & & & & & & & & -0.457 & 0.168 & -0.098 & 0.206 & -0.143 & -0.129 & 0.316 & -0.101 & -0.018 & 0.208 \\
\hline \multirow[t]{2}{*}{ TW } & $\mathrm{rg}_{\mathrm{g}}$ & & & & & & & & & & -0.272 & 0.244 & -0.739 & 0.286 & 0.226 & -0.517 & 0.325 & 0.581 & -0.373 \\
\hline & $r_{p}$ & & & & & & & & & & -0.104 & 0.116 & -0.364 & 0.167 & 0.236 & - & 0172 & 0330 & -0 \\
\hline \multirow[t]{2}{*}{ TB at $F$} & $\mathrm{rg}_{\mathrm{g}}$ & & & & & & & & & & & 0.332 & 0.451 & -0.217 & -0.591 & 0.310 & 0.039 & 0.040 & 0.302 \\
\hline & $r_{p}$ & & & & & & & & & & & 0.313 & 0.388 & -0.197 & -0.296 & 0.272 & 0.040 & 0.034 & 0.275 \\
\hline \multirow[t]{2}{*}{ TB at M } & $\mathrm{r}_{\mathrm{g}}$ & & & & & & & & & & & & 0.225 & 0.250 & 0.178 & -0.032 & 0.237 & 0.140 & 0.109 \\
\hline & $r_{p}$ & & & & & & & & & & & & 0.188 & 0.241 & 0.142 & -0.030 & 0.223 & 0.134 & 0.103 \\
\hline \multirow[t]{2}{*}{ ATR } & $\mathrm{rg}_{\mathrm{g}}$ & & & & & & & & & & & & & 0.363 & 0.146 & 0.072 & 0.238 & -0.285 & 0.320 \\
\hline & $r_{p}$ & & & & & & & & & & & & & 0.346 & 0.125 & 0.070 & 0.222 & -0.271 & 0.307 \\
\hline \multirow[t]{2}{*}{$\begin{array}{l}\text { ACR } \\
\end{array}$} & $\mathrm{rg}_{\mathrm{g}}$ & & & & & & & & & & & & & & 0.451 & -0.224 & 0.201 & 0.134 & -0.341 \\
\hline & $r_{p}$ & & & & & & & & & & & & & & 0.282 & -0.223 & 0.199 & 0.134 & -0.340 \\
\hline \multirow[t]{2}{*}{ MT } & $\mathrm{rg}_{\mathrm{g}}$ & & & & & & & & & & & & & & & 0.036 & 0.048 & 0.106 & -0.305 \\
\hline & $r_{p}$ & & & & & & & & & & & & & & & 0.025 & 0.046 & 0.060 & -0.190 \\
\hline \multirow[t]{2}{*}{ RL } & $\mathrm{rg}_{\mathrm{g}}$ & & & & & & & & & & & & & & & & 0.186 & 0.267 & 0.305 \\
\hline & $\mathrm{r}_{\mathrm{p}}$ & & & & & & & & & & & & & & & & 0.183 & 0265 & 0303 \\
\hline \multirow[t]{2}{*}{ RV } & $\mathrm{rg}_{\mathrm{g}}$ & & & & & & & & & & & & & & & & & 0.424 & -0.038 \\
\hline & $r_{p}$ & & & & & & & & & & & & & & & & & 0.420 & -0.037 \\
\hline RDW & $\mathrm{rg}_{\mathrm{g}}$ & & & & & & & & & & & & & & & & & & -0.465 \\
\hline & $r_{p}$ & & & & & & & & & & & & & & & & & & $\begin{array}{l}-0.464 \\
\end{array}$ \\
\hline
\end{tabular}


Table.2 Estimates of genotypic and phenotypic correlation between morphological characters in rice genotypes in $\mathrm{E}_{2}$

\begin{tabular}{|c|c|c|c|c|c|c|c|c|c|c|c|c|c|c|c|c|c|c|c|}
\hline Characters & Correlation & WP & RWC & PL & FG/P & SG/P & EBT & SY & HI & TW & TB at $F$ & TB at $M$ & ATR & ACR & MT & RL & RV & RDW & GY \\
\hline \multirow[t]{2}{*}{ PH } & $r_{g}$ & 0.237 & 0.475 & 0.024 & 0.574 & -0.221 & 0.989 & 0.316 & -0.012 & 0.084 & 0.259 & 0.525 & 0.239 & 0.112 & -0.372 & 0.247 & -0.210 & 0.301 & 0.330 \\
\hline & $r_{p}$ & 0.191 & 0.390 & 0.018 & 0.443 & -0.177 & 0.427 & 0.257 & -0.052 & 0.011 & 0.171 & 0.427 & 0.180 & 0.089 & -0.281 & 0.200 & -0.170 & 0.246 & 0.266 \\
\hline \multirow[b]{2}{*}{ WP } & $r_{g}$ & & -0.045 & 0.015 & 0.441 & 0.366 & -0.02 & -0.044 & -0.507 & -0.28 & -0.025 & 0.116 & -0.436 & 0.365 & 0.68 & -0.248 & -0.412 & 0.332 & -0.202 \\
\hline & $\frac{r_{p}}{r_{p}}$ & & -0.045 & 0.014 & 0.429 & 0.357 & -0.02 & -0.044 & -0.415 & -0.28 & -0.026 & 0.111 & -0.408 & 0.364 & 0.067 & -0.247 & -0.412 & 0.331 & -0.202 \\
\hline \multirow[b]{2}{*}{ RWC } & $r_{g}$ & & & 0.158 & 0.120 & 0.190 & 0.423 & 0.485 & -0.188 & 0.391 & 0.308 & 0.223 & 0.427 & 0.340 & 0.073 & -0.088 & -0.194 & 0.420 & 0.282 \\
\hline & $\frac{g}{r_{p}}$ & & & 0.157 & 0.115 & 0.183 & 0.226 & $0.484 *$ & -0.157 & 0.290 & 0.276 & 0.219 & 0.400 & 0.340 & 0.071 & -0.088 & -0.194 & 0.419 & 0.281 \\
\hline \multirow[b]{2}{*}{ PL } & $r_{g}$ & & & & 0.104 & -0.570 & -0.14 & 0.753 & 0.566 & 0.228 & 0.586 & 0.466 & 0.077 & -0.036 & -0.862 & -0.142 & -0.164 & -0.095 & 0.631 \\
\hline & $r_{p}$ & & & & 0.096 & $-0.59 *$ & -0.070 & 0.750 *** & 0.457 & 0.172 & $0.526^{*}$ & 0.445 & 0.073 & -0.036 & $-0.833^{*}$ & -0.141 & -0.163 & -0.095 & $0.628 * *$ \\
\hline \multirow[b]{2}{*}{ FG/P } & $r_{g}$ & & & & & -0.137 & -0.016 & 0.212 & -0.082 & -0.01 & 0.231 & 0.482 & -0.305 & 0.165 & -0.310 & 0.014 & -0.212 & 0.436 & -0.149 \\
\hline & $r_{p}$ & & & & & -0.115 & -0.049 & 0.205 & -0.052 & 0.028 & 0.191 & 0.444 & -0.277 & 0.160 & -0.293 & 0.013 & -0.205 & 0.423 & -0.144 \\
\hline \multirow[b]{2}{*}{ SG/P } & $r_{g}$ & & & & & & 0.128 & -0.566 & -0.277 & -0.19 & -0.637 & -0.638 & 0.018 & 0.052 & 0.735 & 0.217 & 0.068 & 0.061 & -0.623 \\
\hline & $r_{p}$ & & & & & & 0.097 & $-0.551^{*}$ & -0.255 & -0.13 & $-0.564 *$ & $-0.606 * *$ & 0.018 & 0.050 & $0.692 * *$ & 0.212 & 0.067 & 0.062 & $-0.604 *$ \\
\hline \multirow[b]{2}{*}{ EBT } & $r_{g}$ & & & & & & & 0.388 & -0.079 & 0.278 & 0.338 & 0.578 & 0.487 & 0.130 & -0.111 & -0.417 & -0.551 & 0.462 & 0.393 \\
\hline & $r_{p}$ & & & & & & & 0.213 & -0.026 & 0.107 & 0.211 & 0.325 & 0.255 & 0.073 & -0.119 & -0.231 & -0.303 & 0.246 & 0.213 \\
\hline \multirow[b]{2}{*}{ SY } & $r_{g}$ & & & & & & & & 0.657 & 0.370 & 0.591 & 0.572 & 0.369 & 0.133 & -0.767 & -0.122 & -0.157 & -0.190 & 0.794 \\
\hline & $r_{p}$ & & & & & & & & $0.537^{*}$ & 0.271 & $0.535^{*}$ & $0.552^{*}$ & 0.347 & 0.133 & $-0.747 *$ & -0.122 & -0.157 & -0.189 & $0.793 * *$ \\
\hline \multirow[b]{2}{*}{ HI } & $\frac{p}{r_{g}}$ & & & & & & & & & 0.268 & 0.382 & 0.129 & 0.372 & 0.053 & -0.801 & 0.348 & 0.327 & -0.453 & 0.495 \\
\hline & $\mathrm{r}_{\mathrm{p}}$ & & & & & & & & & 0.216 & 0.324 & 0.110 & 0.327 & 0.041 & $-0.655^{*}$ & 0.283 & 0.267 & -0.375 & 0.399 \\
\hline \multirow[b]{2}{*}{ TW } & $r_{g}$ & & & & & & & & & & 0.433 & 0.444 & 0.072 & 0.189 & -0.355 & -0.259 & 0.282 & 0.292 & 0.339 \\
\hline & $r_{p}$ & & & & & & & & & & 0.331 & 0.315 & 0.044 & 0.139 & -0.246 & -0.192 & 0.209 & 0.217 & 0.257 \\
\hline \multirow[b]{2}{*}{ TB at $F$} & $\mathrm{r}_{g}$ & & & & & & & & & & & 0.837 & 0.430 & -0.113 & -0.919 & -0.136 & -0.099 & 0.044 & 0.645 \\
\hline & $r_{p}$ & & & & & & & & & & & $0.718^{* * *}$ & 0.393 & -0.104 & $-0.804 *$ & -0.123 & -0.092 & 0.038 & $0.581 *$ \\
\hline \multirow[b]{2}{*}{ TB at $M$} & $r_{g}$ & & & & & & & & & & & & -0.045 & 0.029 & -0.767 & -0.321 & -0.213 & 0.268 & 0.628 \\
\hline & $r_{p}$ & & & & & & & & & & & & -0.038 & 0.028 & $-0.732 *$ & -0.310 & -0.205 & 0.258 & $0.604 * *$ \\
\hline \multirow[b]{2}{*}{ ATR } & $r_{g}$ & & & & & & & & & & & & & -0.261 & -0.088 & 0.224 & 0.061 & -0.382 & 0.444 \\
\hline & $r_{p}$ & & & & & & & & & & & & & -0.246 & -0.088 & 0.210 & 0.057 & -0.360 & 0.416 \\
\hline \multirow[b]{2}{*}{ ACR } & $r_{g}$ & & & & & & & & & & & & & & -0.048 & -0.096 & -0.175 & 0.374 & -0.018 \\
\hline & $r_{p}$ & & & & & & & & & & & & & & -0.046 & -0.096 & -0.175 & 0.373 & -0.019 \\
\hline \multirow[b]{2}{*}{ MT } & $r_{g}$ & & & & & & & & & & & & & & & 0.124 & 0.055 & 0.188 & -0.869 \\
\hline & $r_{p}$ & & & & & & & & & & & & & & & 0.121 & 0.053 & 0.184 & $-0.846^{*}$ \\
\hline \multirow[b]{2}{*}{ RL } & $r_{g}$ & & & & & & & & & & & & & & & & 0.651 & -0.028 & -0.073 \\
\hline & $r_{p}$ & & & & & & & & & & & & & & & & $0.650^{* * *}$ & -0.028 & -0.072 \\
\hline \multirow[b]{2}{*}{ RV } & $r_{g}$ & & & & & & & & & & & & & & & & & 0.039 & 0.003 \\
\hline & $r_{p}$ & & & & & & & & & & & & & & & & & 0.039 & 0.003 \\
\hline \multirow[b]{2}{*}{ RDW } & $r_{g}$ & & & & & & & & & & & & & & & & & & -0.308 \\
\hline & $\frac{r_{p}}{r_{p}}$ & & & & & & & & & & & & & & & & & & -0.307 \\
\hline
\end{tabular}


Table.3 Direct and indirect effects of physio-morphological traits on GY/P at genotypic and phenotypic level in $\mathrm{E}_{1}$

\begin{tabular}{|c|c|c|c|c|c|c|c|c|c|c|c|c|c|c|c|c|c|c|c|}
\hline Character & Env. & PH & WP & RWC & $\mathbf{P L}$ & FG/P & SG/P & EBT & SY & HI & TW & TB at $\mathbf{F}$ & TB at $M$ & ATR & ACR & MT & RL & RV & RDW \\
\hline \multirow[t]{2}{*}{ PH } & $E_{1}$ & -1.47 & 1.152 & 0.121 & -0.004 & -0.484 & -0.031 & 0.455 & 0.036 & -0.024 & -0.049 & 0.207 & -0.112 & 0.137 & 0.000 & 0.004 & 0.419 & -0.037 & -0.321 \\
\hline & $\mathrm{E}_{2}$ & 0.647 & 0.372 & -0.022 & 0.011 & -0.413 & -0.007 & -0.023 & 0.036 & -0.119 & 0.013 & 0.069 & -0.202 & 0.040 & 0.00 & -0.004 & 0.00 & -0.135 & -0.270 \\
\hline \multirow[t]{2}{*}{ WP } & $E_{1}$ & & 2.581 & -0.193 & 0.003 & -0.338 & 0.095 & -0.483 & 0.095 & -0.045 & -0.156 & -0.045 & -0.191 & -0.123 & -0.011 & 0.008 & -0.13 & -0.033 & -0.459 \\
\hline & $E_{2}$ & & 0.999 & 0.041 & -0.017 & -0.327 & 0.021 & 0.032 & 0.112 & -0.216 & 0.057 & -0.019 & -0.400 & -0.042 & -0.007 & 0.006 & 0.00 & -0.093 & -0.461 \\
\hline \multirow[t]{2}{*}{ RWC } & $E_{1}$ & & & 0.541 & -0.033 & 0.071 & -0.035 & 0.954 & -0.154 & -0.029 & -0.075 & -0.103 & 0.049 & 0.047 & -0.053 & 0.010 & -0.29 & 0.031 & -0.130 \\
\hline & $\mathrm{E}_{2}$ & & & -0.116 & 0.187 & 0.070 & -0.008 & -0.062 & -0.180 & -0.136 & 0.027 & -0.044 & -0.103 & 0.016 & -0.035 & 0.007 & 0.00 & 0.090 & -0.131 \\
\hline \multirow[t]{2}{*}{$\mathbf{P L}$} & $E_{1}$ & & & & 0.112 & 0.105 & -0.193 & -0.440 & 0.318 & 0.038 & 0.028 & -0.070 & 0.017 & 0.101 & 0.046 & 0.037 & 0.110 & 0.023 & 0.005 \\
\hline & $E_{2}$ & & & & -0.638 & 0.099 & -0.043 & 0.033 & 0.368 & 0.183 & -0.012 & -0.028 & 0.035 & 0.033 & 0.030 & 0.026 & 0.00 & 0.064 & 0.006 \\
\hline \multirow[t]{2}{*}{ FG/P } & $E_{1}$ & & & & & $\begin{array}{l}-0.721 \\
\end{array}$ & 0.057 & 0.212 & 0.185 & -0.013 & -0.035 & 0.106 & -0.088 & -0.069 & -0.047 & -0.005 & 0.073 & -0.098 & 0.066 \\
\hline & $E_{2}$ & & & & & -0.752 & 0.013 & -0.016 & 0.206 & -0.087 & 0.018 & 0.046 & -0.179 & 0.023 & -0.028 & -0.001 & 0.00 & -0.268 & 0.059 \\
\hline \multirow[t]{2}{*}{ SG/P } & $E_{1}$ & & & & & & 0.586 & -0.176 & -0.175 & -0.019 & -0.048 & -0.180 & 0.062 & -0.441 & -0.039 & -0.012 & 0.115 & 0.012 & -0.622 \\
\hline & $E_{2}$ & & & & & & 0.136 & 0.010 & -0.200 & -0.068 & 0.015 & -0.081 & 0.127 & -0.146 & -0.024 & -0.006 & 0.00 & -0.036 & -0.613 \\
\hline \multirow[t]{2}{*}{ EBT } & $E_{1}$ & & & & & & & 1.137 & -0.294 & 0.000 & 0.011 & 0.099 & 0.099 & 0.268 & -0.022 & -0.020 & -0.19 & -0.005 & 0.307 \\
\hline & $\mathrm{E}_{2}$ & & & & & & & -0.103 & -0.246 & -0.001 & 0.025 & 0.013 & 0.140 & 0.068 & -0.010 & -0.008 & 0.00 & -0.012 & 0.224 \\
\hline \multirow[t]{2}{*}{ SY } & $E_{1}$ & & & & & & & & 0.595 & 0.001 & 0.033 & 0.018 & -0.023 & 0.054 & 0.011 & 0.030 & 0.140 & -0.116 & 0.400 \\
\hline & $\mathrm{E}_{2}$ & & & & & & & & 0.701 & 0.001 & -0.010 & 0.006 & -0.051 & 0.018 & 0.007 & 0.022 & 0.00 & -0.334 & 0.403 \\
\hline \multirow[t]{2}{*}{ HI } & $E_{1}$ & & & & & & & & & 0.114 & 0.160 & 0.188 & 0.038 & 1.012 & 0.036 & -0.027 & 0.458 & -0.038 & 0.025 \\
\hline & $E_{2}$ & & & & & & & & & 0.676 & -0.059 & 0.050 & 0.067 & 0.035 & 0.019 & -0.009 & 0.00 & -0.091 & 0.020 \\
\hline \multirow[t]{2}{*}{ TW } & $E_{1}$ & & & & & & & & & & -0.197 & -0.172 & -0.076 & -0.348 & -0.058 & 0.015 & -0.60 & 0.101 & -0.667 \\
\hline & $E_{2}$ & & & & & & & & & & 0.129 & -0.031 & -0.079 & -0.061 & -0.022 & 0.017 & 0.00 & 0.156 & -0.383 \\
\hline \multirow[t]{2}{*}{ TB at $F$} & $E_{1}$ & & & & & & & & & & & 0.632 & -0.103 & 0.212 & 0.044 & -0.038 & 0.359 & 0.012 & -0.046 \\
\hline & $\mathrm{E}_{2}$ & & & & & & & & & & & 0.299 & -0.211 & 0.064 & 0.026 & -0.021 & 0.00 & 0.036 & -0.040 \\
\hline \multirow[t]{2}{*}{ TB at M } & $E_{1}$ & & & & & & & & & & & & -0.310 & 0.106 & -0.050 & 0.011 & -0.04 & 0.074 & -0.160 \\
\hline & $E_{2}$ & & & & & & & & & & & & $\begin{array}{l}-0.679 \\
\end{array}$ & 0.032 & -0.031 & 0.010 & 0.00 & 0.202 & -0.155 \\
\hline \multirow[t]{2}{*}{ ATR } & $E_{1}$ & & & & & & & & & & & & & 0.471 & -0.073 & 0.009 & 0.083 & 0.074 & 0.327 \\
\hline & $E_{2}$ & & & & & & & & & & & & & 0.168 & -0.045 & 0.009 & 0.00 & 0.201 & 0.315 \\
\hline \multirow[t]{2}{*}{ ACR } & $\mathrm{E}_{1}$ & & & & & & & & & & & & & & -0.201 & 0.029 & -0.26 & 0.062 & -0.153 \\
\hline & $\mathrm{E}_{2}$ & & & & & & & & & & & & & & -0.131 & 0.020 & 0.00 & 0.180 & -0.155 \\
\hline \multirow[t]{2}{*}{ MT } & $\mathrm{E}_{1}$ & & & & & & & & & & & & & & & 0.065 & 0.042 & 0.015 & -0.122 \\
\hline & $\mathrm{E}_{2}$ & & & & & & & & & & & & & & & 0.072 & 0.00 & 0.042 & -0.070 \\
\hline \multirow[t]{2}{*}{$\mathbf{R L}$} & $E_{1}$ & & & & & & & & & & & & & & & & 1.158 & 0.058 & -0.306 \\
\hline & $E_{2}$ & & & & & & & & & & & & & & & & 0.02 & 0.166 & -0.308 \\
\hline \multirow[t]{2}{*}{$\mathbf{R V}$} & $E_{1}$ & & & & & & & & & & & & & & & & & 0.310 & -0.486 \\
\hline & $\mathrm{E}_{2}$ & & & & & & & & & & & & & & & & & 0.906 & -0.487 \\
\hline \multirow[t]{2}{*}{ RDW } & $E_{1}$ & & & & & & & & & & & & & & & & & & -1.147 \\
\hline & $\mathrm{E}_{2}$ & & & & & & & & & & & & & & & & & & -1.160 \\
\hline
\end{tabular}

Residual effects $=0.226$, Bold figures indicate direct effects. 
Table.4 Direct and indirect effects of physio-morphological traits on GY/P at genotypic and phenotypic level in $\mathrm{E}_{2}$

\begin{tabular}{|c|c|c|c|c|c|c|c|c|c|c|c|c|c|c|c|c|c|c|c|}
\hline Character & Env. & PH & WP & RWC & $\mathbf{P L}$ & FG/P & SG/P & EBT & SY & HI & TW & TB at $F$ & TB at M & ATR & ACR & MT & RL & RV & RDW \\
\hline \multirow[t]{2}{*}{ PH } & $\mathrm{E}_{1}$ & 0.851 & -0.032 & 0.406 & 0.007 & -0.317 & -0.022 & 0.029 & -0.092 & -0.001 & -0.085 & 0.025 & 0.145 & -0.078 & 0.023 & 0.231 & -0.292 & -0.292 & -0.178 \\
\hline & $\mathrm{E}_{2}$ & 0.243 & -0.028 & -0.025 & 0.014 & -0.041 & -0.008 & 0.079 & 0.00 & 0.020 & 0.00 & 0.009 & 0.278 & 0.039 & 0.042 & -0.071 & -0.022 & -0.090 & -0.173 \\
\hline \multirow[t]{2}{*}{ WP } & $E_{1}$ & & -0.134 & -0.039 & 0.004 & -0.243 & 0.036 & -0.001 & 0.013 & -0.052 & 0.285 & -0.002 & 0.032 & 0.142 & 0.073 & -0.042 & 0.293 & -0.574 & -0.196 \\
\hline & $\mathrm{E}_{2}$ & & -0.147 & 0.003 & 0.011 & -0.040 & -0.016 & -0.003 & 0.00 & 0.156 & 0.007 & -0.001 & 0.072 & -0.089 & 0.175 & 0.017 & 0.027 & -0.219 & -0.232 \\
\hline \multirow[t]{2}{*}{ RWC } & $E_{1}$ & & & $\mathbf{0 . 8 5 5}$ & 0.048 & -0.066 & 0.019 & 0.013 & -0.142 & -0.119 & -0.397 & 0.030 & 0.061 & -0.139 & 0.069 & -0.046 & 0.104 & -0.270 & -0.248 \\
\hline & $\mathrm{E}_{2}$ & & & -0.065 & 0.119 & -0.011 & 0.008 & 0.042 & 0.001 & 0.059 & -0.010 & 0.014 & 0.143 & 0.088 & 0.163 & 0.018 & 0.010 & -0.103 & -0.294 \\
\hline \multirow[t]{2}{*}{ PL } & $E_{1}$ & & & & $\mathbf{0 . 3 0 7}$ & -0.057 & -0.056 & -0.004 & -0.220 & 0.058 & -0.232 & 0.057 & 0.128 & -0.025 & -0.007 & 0.535 & 0.167 & -0.228 & 0.056 \\
\hline & $E_{2}$ & & & & 0.758 & -0.009 & -0.024 & -0.013 & 0.001 & -0.172 & -0.006 & 0.027 & 0.290 & 0.016 & -0.017 & -0.211 & 0.015 & -0.087 & 0.067 \\
\hline \multirow[t]{2}{*}{ FG/P } & $E_{1}$ & & & & & -0.551 & -0.014 & 0.00 & -0.062 & -0.008 & 0.012 & 0.022 & 0.133 & 0.099 & 0.033 & 0.192 & -0.017 & -0.294 & -0.257 \\
\hline & $\mathrm{E}_{2}$ & & & & & -0.093 & -0.005 & -0.009 & 0.00 & 0.020 & -0.001 & -0.010 & 0.289 & 0.061 & 0.077 & -0.074 & -0.001 & -0.109 & -0.297 \\
\hline \multirow[t]{2}{*}{ SG/P } & $E_{1}$ & & & & & & 0.099 & 0.004 & 0.166 & -0.028 & 0.198 & -0.062 & -0.176 & -0.006 & 0.010 & -0.457 & -0.257 & 0.095 & -0.036 \\
\hline & $E_{2}$ & & & & & & 0.044 & 0.018 & -0.001 & 0.096 & 0.005 & -0.029 & -0.395 & 0.004 & 0.024 & 0.175 & -0.023 & 0.036 & -0.044 \\
\hline \multirow[t]{2}{*}{ EBT } & $E_{1}$ & & & & & & & 0.030 & -0.114 & -0.008 & -0.282 & 0.033 & 0.159 & -0.159 & 0.026 & 0.069 & 0.493 & -0.766 & -0.273 \\
\hline & $E_{2}$ & & & & & & & 0.185 & 0.00 & 0.010 & -0.004 & 0.011 & 0.212 & 0.056 & 0.035 & -0.030 & 0.025 & -0.161 & -0.173 \\
\hline \multirow[t]{2}{*}{ SY } & $E_{1}$ & & & & & & & & \begin{tabular}{|c|c|} 
\\
\end{tabular} & 0.067 & -0.375 & 0.057 & 0.158 & -0.120 & 0.027 & 0.476 & 0.144 & -0.219 & 0.112 \\
\hline & $\mathrm{E}_{2}$ & & & & & & & & $\mathbf{0 . 0 0 2}$ & -0.202 & -0.009 & 0.028 & 0.360 & 0.076 & 0.064 & -0.189 & 0.013 & -0.084 & 0.133 \\
\hline \multirow[t]{2}{*}{ HI } & $E_{1}$ & & & & & & & & & $\mathbf{0 . 1 0 2}$ & -0.272 & 0.037 & 0.036 & -0.121 & 0.011 & 0.498 & -0.412 & 0.455 & 0.267 \\
\hline & $E_{2}$ & & & & & & & & & -0.376 & -0.008 & 0.017 & 0.071 & 0.072 & 0.020 & -0.166 & -0.031 & 0.142 & 0.263 \\
\hline \multirow[t]{2}{*}{ TW } & $\mathrm{E}_{1}$ & & & & & & & & & & -1.014 & 0.042 & 0.122 & -0.023 & 0.038 & 0.220 & 0.306 & 0.392 & -0.172 \\
\hline & $E_{2}$ & & & & & & & & & & $-\mathbf{- 0 . 0 3 5}$ & 0.017 & 0.206 & 0.010 & 0.067 & -0.062 & 0.021 & 0.111 & -0.152 \\
\hline \multirow[t]{2}{*}{ TB at F } & $\mathrm{E}_{1}$ & & & & & & & & & & & 0.097 & 0.231 & -0.140 & -0.023 & 0.571 & 0.161 & -0.138 & -0.026 \\
\hline & $\mathrm{E}_{2}$ & & & & & & & & & & & 0.052 & 0.468 & 0.086 & -0.050 & -0.204 & 0.014 & -0.049 & -0.027 \\
\hline \multirow[t]{2}{*}{ TB at M } & $\mathrm{E}_{1}$ & & & & & & & & & & & & 0.275 & 0.015 & 0.006 & 0.477 & 0.380 & -0.296 & -0.158 \\
\hline & $E_{2}$ & & & & & & & & & & & & 0.652 & -0.008 & 0.013 & -0.185 & 0.034 & -0.109 & -0.181 \\
\hline \multirow[t]{2}{*}{ ATR } & $\mathrm{E}_{1}$ & & & & & & & & & & & & & -0.326 & -0.053 & 0.055 & -0.265 & 0.085 & 0.225 \\
\hline & $E_{2}$ & & & & & & & & & & & & & 0.219 & -0.118 & -0.022 & -0.023 & 0.030 & 0.253 \\
\hline \multirow[t]{2}{*}{ ACR } & $E_{1}$ & & & & & & & & & & & & & & 0.201 & 0.030 & 0.113 & -0.244 & -0.221 \\
\hline & $\mathrm{E}_{2}$ & & & & & & & & & & & & & & 0.479 & -0.012 & 0.011 & -0.093 & -0.262 \\
\hline \multirow[t]{2}{*}{ MT } & $\mathrm{E}_{1}$ & & & & & & & & & & & & & & & -0.621 & -0.147 & 0.077 & -0.111 \\
\hline & $E_{2}$ & & & & & & & & & & & & & & & $\begin{array}{l}0.253 \\
\end{array}$ & -0.013 & 0.028 & -0.129 \\
\hline \multirow[t]{2}{*}{$\mathbf{R L}$} & $E_{1}$ & & & & & & & & & & & & & & & & -1.183 & 0.905 & 0.017 \\
\hline & $E_{2}$ & & & & & & & & & & & & & & & & -0.110 & 0.346 & 0.020 \\
\hline \multirow[t]{2}{*}{$\mathbf{R V}$} & $E_{1}$ & & & & & & & & & & & & & & & & & 1.392 & -0.023 \\
\hline & $\mathrm{E}_{2}$ & & & & & & & & & & & & & & & & & 0.532 & -0.027 \\
\hline \multirow[t]{2}{*}{ RDW } & $\mathrm{E}_{1}$ & & & & & & & & & & & & & & & & & & -0.590 \\
\hline & $E_{2}$ & & & & & & & & & & & & & & & & & & $\begin{array}{l}-0.702 \\
\end{array}$ \\
\hline
\end{tabular}

Residual effects $=-0.147$ (genotypic) and 0.0621 (phenotypic), Bold figures indicate direct effects. 


\section{Path-coefficient analysis}

In present study, path-coefficient analysis was carried out at phenotypic and genotypic level to assess the direct and indirect effects of component characters on GY/P in E1 and E2. In case of physio-morphological traits, WP, $\mathrm{SY}$ and RV in E1 emerged as most important direct contributors of GY owing the their high order positive direct effects on grain yield at phenotypic as well as genotypic levels. In addition to these, $\mathrm{PH}, \mathrm{HI}$ at phenotypic level and RWC, SG/P, EBT, TB at flowering, ATR and RL at genotypic level extended high order positive direct effects on grain yield in irrigated condition to appear as direct components of secondary importance. Similarly, total biomass at re-watering and ACR at phenotypic level were identified as direct contributors of secondary importance. Plant height, fertile grains per panicle, test weight, total biomass at re-watering and root dry weight via water potential; panicle length via straw yield, straw yield and ATR via root dry weight exerted substantial positive indirect effects on grain yield at phenotypic and genotypic levels to appear as important indirect contributors through different characters in the control condition. In stress condition, straw yield, harvest index, total biomass at maturity and total biomass at flowering appeared most important indirect components of grain yield due to their high order positive indirect effects at phenotypic or genotypic levels through more than one character (Tables 1-4).

The estimates of direct and indirect effects of several of physio-morphological traits at genotypic and phenotypic levels in stress condition were strikingly different in sign and magnitude than the corresponding estimates in control condition. The identify characters exhibiting high estimates of direct and indirect effects in either negative or positive direction at phenotypic and genotypic levels was vastly different in the control and stress (drought) condition. This indicated that expression of grain yield requires different balance physio-morphological traits in drought stress condition than the normal control conditions. Therefore, for devising the selection criteria or index for evolving high yielding genotypes for drought stress environments, the inter-relationships and path effects existing in the stress condition should be given due consideration. The substantial differences in correlations and direct and indirect path effects observed at phenotypic and genotypic levels in control and stress environments in case of physiomorphological traits emphasized the importance of genotypic $\mathrm{x}$ environment interactions in conditioning the interrelationship among various physiomorphological characters in rice. The physiomorphological characters identified as important direct and indirect yield contributing traits in normal and stress conditions, as discussed before, should be given due consideration in formulation of selection strategy aimed at developing high yield rice genotypes for respective environments.

\section{References}

Arnon, D.I. 1949. Copper enzyme in isolated chloroplast: Polyphenol oxidase in Beta vulgaris. Plant Physiol., 24: 1-15.

Asada, K., Takahasi, S.M., Nagate, M. 1974. Assay and inhibition of spinach Superoxide dismutase. Agric. Biol. Chem., 38(2): 171-173.

Babu, C.R., Nguyen, B.D., Varapong, Chamarerk, P., Jeyaprakash, Ganesh, S.K., Palchamy, A., Sadasivam, S., Sarkarung, S., Wade, L.J., Nguyen, H.T. 2003. Genetic analysis of drought tolerance in rice by molecular markers: association between secondary traits and yield performance. Crop Sci., 43: 14571469.

Bahar, B., Yildirim, M. 2010. Heat and drought resistance criteria in spring bread wheat: Drought resistance parameters. Sci. Res. Essays, 5(13): 1742-1745. 
Bai, N.R., Devika, R., Regind, A., Joseph, C.A. 1992. correlation of yield and yield components in medium duration rice cultivars. Environ. Ecol., 10: 469-470.

Bates, L.S., Warden, R.P., Teare, I.D. 1973. Rapid determination of free proline for water stress studies. Plant Soil 39: 205207.

Bayoumi, T.Y., Eid, M.H., Metwali, E.M. 2008. Application of physiological and biochemical indices as a screening technique for drought tolerance in wheat genotypes. Afr. J. Biotechnol., 7(14): 2341-2352.

Bernier, J., Atlin, G.N., Rachid, S., Kumar, A., Spaner, D. 2008. Breeding upland rice for drought resistance. J. Sci. Food Agric., 88: 927-939.

Blum, A. 1988. Plant breeding for stress environments. CRC Press, Boca Raton, FL.

Chance, B., Maechly, A.C. 1955. Assay of catalase and peroxidase, In: Colowick SP and Kaplan NO (eds.), Methods in Enzymology 76-Academic Press, New York.

Chauhan, J.S., Tyagi, M.K., Kumar, A., Nashaat, N.I., Singh, M., Singh, N.B., Jakhar, M.L., Welham, S.J. 2007. Drought effects on yield and its components in Indian mustard (Brassica juncea L). Plant Breed., 126: 399-402.

Fisher, R.A., Maurer, R. 1978. Drought resistance in spring wheat cultivars. I Grain yield response. Aust. J. Agric. Res., 29: 897-912.

Fukai, S., Cooper, M. 1995. Development of drought resistant cultivars using physiomorphological traits in rice. Field Crops Res., 40: 67-86.

Fukai, S., Inthapan, P. 1988. Growth and yield of rice cultivars under sprinkler irrigation in south-eastern Queensland. 3. Water extraction and plant water relationscomparison with maize and grain sorghum. Aust. J. Exp. Agric., 28: 249252.

Gaff, D.F. 1980. Adaptation of Plants to Water and High Temperature Stress (eds Turner, N. C. and Kramer, P. J.), Wiley, New York, 1980. pp. 207-230.
Girish, T.N., Gireesha, T.M., Vaishali, M.G., Hanamareddy, B.G., Hittalmani, S. 2006. Response of new IR 50/ Morobereken recombinant inbred population of rice (Oryza sativa L.) from an indica $\mathrm{x}$ japonica cross for growth and yield traits under arobic condition. Euphytica, 152(2): 149-161.

Gomez, K.A., Gomez, A.A. 1984. Statistical Procedures for Agricultural Research (2nd Edition. New York (USA) John Wiley and Sons. Inc. 680 p.

IRRI. 1996. Standard Evaluation System for Rice (4th Edition. International Rice Testing Programme, International Rice Research institute, Los Banos, Philippines.

IRRI. 2009. Crop Stat 7.2 for Windows. Crop Research Informatics Laboratory, International Rice Research Institute, Los Banos, Philippines.

Jowarski, K. 1971. Nitrate reductase assay for nitrite reductase in barley aleurone layer. Plant Physiol., 47: 790-794.

Khush, G.S. 1997. Origin, dispersal, cultivation and variation of rice. Plant Mol. Biol., 35: 25-34.

Laffite, H.R. 2002. Relationship between leaf relative water content during reproductive stage water deficit and grain formation in rice. Field Crop Res., 76: 165-174.

Levitt, J. 1972. Responses of Plants to Environmental Stresses, Academic Press, New York.

Lowery, O.H., Rosenbrough, N.J., Farr, A.L., Randall, R.J. 1951. Protein measurement with proline phenol reagent. J. Biol. Chem., 193: 265-275.

Makara, O., Basnayake, J., Tsubo, M., Fukai, S., Fischer, K.S., Cooper, M., Nesbitt, H. 2006. Use of drought response index for identification of drought tolerant genotypes in rainfed lowland rice. Field Crop Res., 99: 48-58.

Mall, A.K., Swain, P., Singh, O.N. 2011. Genetic divergence studies in drought promising rice genotypes based on quality characters. Indian J. Plant Genetic Res., 24(2): 172-176.

Mc Cready, R.M., Guggols, J., Silviers, V., Owen, H.S. 1950. Determination of starch 
and amylase in vegetables. Ann. Chem., 22: $1156-1158$.

Mitra, J. 2001. Genetics and genetic improvement of drought resistance in crop plants. Curr. Sci., 80(6): 758-763.

Nguyen, H.T., Babu, R.C., Blum, A. 1997. Breeding for drought resistance in rice: Physiology and molecular genetics considerations. Crop Sci., 37: 1426-1434.

O'Toole, J.C., Namuco, O.S. 1983. Role of panicle exsertion in water stress induced sterility. Crop Sci., 23: 1093-1097.

Parameshwarappa, S.G., Salimath, P.M. 2008. Field Screening of Chickpea Genotypes for Drought Resistance. Karnataka J. Agric. Sci., 21(1): 113-114.

Puri, R.R., Khadka, K., Paudyal, A. 2010. Separating climate resilient crops through screening of drought tolerant rice land races in Nepal. Agron. J. Nepal, 1: 80-84.

Raman, A., Verulkar, S.B., Mandal, N.P., Variar, M., Shukla, V.D., Dwivedi, J.L., Singh, B.N., Singh, O.N., Swain, P., Mall, A.K., Robin, S., Chandrababu, R., Jain, A., Ram, T., Hittalmani S, Haefele S, Piepho HP, Kumar, A. 2012. Drought yield index to select high yielding rice lines under different drought stress severities. Rice, 5: 31.

Reddy, Y.A.N., Prasad, T.G., Kumar, M.U. 1995. Genotypic variation for yield attributes and yield of rice. Madras Agric. J., 82(4): 310-313.

Reyniers, F.N., Troung-Binh, L., Jacquinot, Nicou, R. 1982. Breeding for drought resistance in dryland rice. P. 273-292. In Drought Resistance in Crops with Emphasis on Rice. Int. Rice Res. Inst., Los Banos, Philippines.
Saadalla, M.M., Shanahan, J.F,. Quick, J.S. 1990. Heat tolerance in winter wheat I. Hardening and genetic effects on membrane thermo stability. Crop Sci., 30: 1243-1247.

Samadia, D.K. 2005. Genetic variability studies in Lasora (Cordia myxa Roxb.. Indian J. Plant Genet. Res., 18(3): 236-240.

Singh, R.K., Chaudhury, B.D. 1985 Biometrical Methods in Quantitative Genetic Analysis. Kalyani Publishers, New Delhi, India.

Valliyodan, B., Nguyen, H.T. 2006. Understanding regulatory networks and engineering for enhanced drought tolerance in plants. Curr. Opinion in Plant Biol., 9: 1-7.

Wade, L.J., McLaren, C.G., Quintana, L., Harnpichitvitaya, D., Rajatasereekul, S., Sarawgi, A.K., Kumar, A., Ahmed, H.U.S., Singh, A.K., Rodriguez, R,. Siopongco, J., Sarkarung, S. 1999. Genotype by environment interactions across diverse rainfed lowland rice environments. Field Crops Res., 64: 3550.

Weatherley, P.E. 1965. In the state and movement of water in living organisms. In: G.E. Fogg. (ed.), University Press London and New York, pp. 157-184.

Yamm, E.W., Willis, A.J. 1954. The estimation of carbohydrates in plant extracts by anthrone. Biochem. J., 57: 508-514.

Yoshida, S., Ahn, S.B. 1968. The accumulation of carbohydrate in rice varieties in relation to their response to nitrogen in the tropics. Soil Sci. Plant Nutr., 14(4): 103-112.

\section{How to cite this article:}

Singh, A.K. and Mall, A.K. 2017. Determine Physiological Traits Associated with Flowering Stage Drought Tolerance in Lowland Rice (Oryza sativa L.) genotypes. Int.J.Curr.Microbiol.App.Sci. 6(6): 910-920. doi: https://doi.org/10.20546/ijcmas.2017.606.107 